$17^{\text {th }}$ International Congress of Metrology, 02012 (2015)

DOI: $10.1051 /$ metrology / 201502012

(C) Owned by the authors, published by EDP Sciences, 2015

\title{
A user-friendly software for a simple and validated implementation of GUM Supplement 1
}

\author{
Géraldine Ebrard $^{1, a}$, Alexandre Allard ${ }^{1}$ et Nicolas Fischer $^{1}$ \\ ${ }^{1}$ Laboratoire national de métrologie et d'essais, Trappes, France
}

\begin{abstract}
Résumé. Afin d'appliquer la méthode d'évaluation de l'incertitude de Monte Carlo [1] nécessitant des ressources informatiques pour la génération des nombres aléatoires, le logiciel LNE-MCM a été développé. Facile à prendre en main, doté d'un générateur de nombres pseudo-aléatoires de qualité, il est dédié à l'évaluation de l'incertitude de mesure par simulations de Monte Carlo. Il permet, en outre, de traiter le cas de modèles multivariés, de réaliser une analyse de sensibilité et de procéder à des tests d'adéquation. Le logiciel LNE-MCM sera mis en ligne gratuitement fin 2015.
\end{abstract}

\section{Introduction}

To evaluate the uncertainty in measurement, one has commonly used the well-known "Guide to the expression of Uncertainty in Measurement" Uncertainty Framework (GUF). This method described in the "Guide to the expression of Uncertainty in Measurement" (GUM) is based on the Law of Propagation of Uncertainty (LPU) [2]. However the conditions for the use of the GUM Uncertainty Framework are not fulfilled for a lot of common cases. For example when the linearization of the measurement model provides an inadequate representation, the GUF will lead to an unreliable estimation of the standard uncertainty associated with the output quantity. To account for this limitation, one has to adopt a method with a broader scope, such as the method described in the Supplement 1 to the GUM [1] and concerned with the implementation of the propagation of distributions. This alternative to the LPU method, commonly called the Monte Carlo Method, is a stochastic procedure consisting of making random draws from probability density functions (PDF) for the input quantities in order to compute a sample for the output quantity. A pseudo-random number generator is required to this extent. Therefore we developed a user-friendly software called LNE-MCM. It is implemented in Matlab language and dedicated to the evaluation of the measurement uncertainty using Monte Carlo simulations. Pseudo-random number generator is taken from Mersenne Twister's work [3]. We specify that the measurement models must be explicit. Besides, this software enables the users to choose between several probability distributions to represent the uncertainty of the input quantities. Correlations between input quantities are also taken into account when their marginal distributions are either Gaussian or Student. Moreover, LNE-MCM software is able to deal with more than one output quantity as explained in the GUM Supplement 2 [4]. Beyond the scope of GUM Supplement 1, LNEMCM software provides an uncertainty budget based on a sensitivity analysis. Two methods are available: the Spearman correlation coefficient for monotonic measurement models and Sobol' indices for more complex models [5]. The Kolmogorov-Smirnov goodness of fit test can be used to determine the probability distribution which is the closest to the obtained empirical
PDF [6]. Finally, a numerical validation is provided to check whether the GUF is valid as recommended in the GUM Supplement 1. Illustration of LNE-MCM software will be based on the example of the GUM Supplement 2: the resistance and reactance of a circuit element [4] as shown in figure 1.

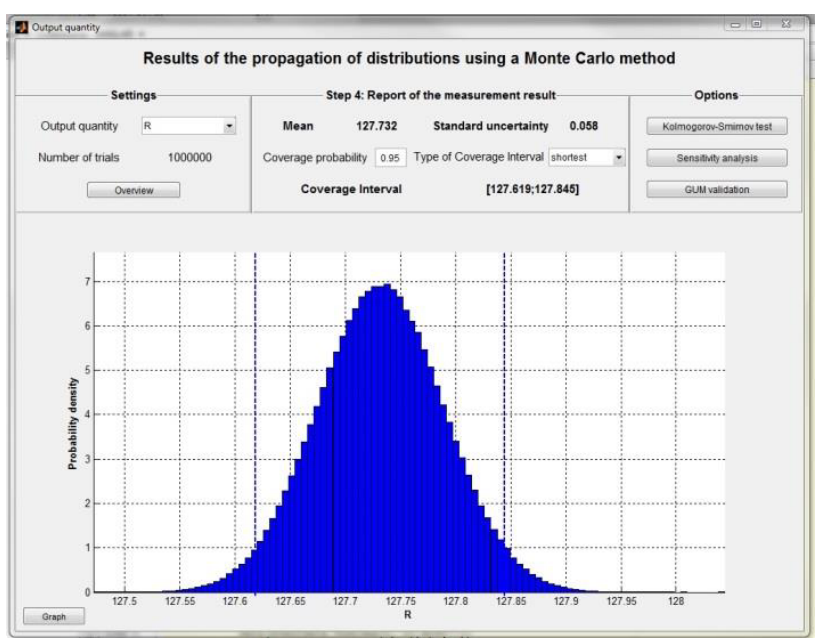

Figure 1. Results for the output quantity R given by LNEMCM software

In the following sections, we summarize the main principles of the Monte Carlo Method and we describe the additional features added in the LNE-MCM software.

\section{Monte Carlo Method (GUM S1)}

As mentioned before, the Monte Carlo Method is an alternative method to the LPU when conditions to apply the LPU method are not fulfilled [1,7]. Indeed, within the GUF, the estimation of uncertainty in measurement is generally based on a first-order Taylor development, which is not correct in case of strongly non-linear models. Additionally, when the PDF for the output quantity departs appreciably from a Gaussian distribution, coverage intervals are unrealistic. On the contrary, the Monte Carlo Method provides coverage intervals which require no assumptions on the distribution of the output quantity. The Monte Carlo

\footnotetext{
${ }^{\mathrm{a}}$ Corresponding author : Geraldine.Ebrard@lne.fr
}

This is an Open Access article distributed under the terms of the Creative Commons Attribution License 4.0, which permits unrestricted use, distribution, and reproduction in any medium, provided the original work is properly cited. 
Method can be summarized in four main steps as illustrated in figure 2. In the first step, the measurement process is analysed in order to precisely define the measurand, list the input quantities and also determine the measurement model. Then, thanks to prior information, PDF are assigned to the input quantities. In the third step, the distributions are propagated through the mathematical model using Monte Carlo simulations. Finally, the measurement result is reported using statistical parameters such as the mean, the standard deviation and quantiles defining a coverage interval. In LNE-MCM software, we assume that the analysis of the measurement process is done before use so that the mathematical model, the input quantities and their PDF are known. Then the user is guided all the time in use by following the previous steps.

In order to widen the field of possibilities and by the same way increase the interest of the users, additional features were implemented that go beyond the GUM Supplement 1.

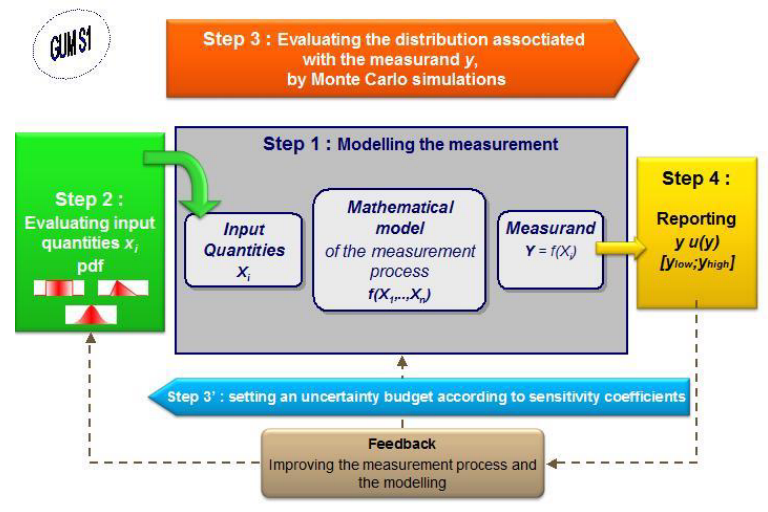

Figure 2. Summary of procedure for evaluating and reporting uncertainty.

\section{Additional features}

Indeed, LNE-MCM enables the user to consider measurement models with any number of output quantities [4], to perform a sensitivity analysis with two methods: Spearman rank correlation and Sobol' indices [5], to realize goodness-of-fit test [6] and to validate the assumptions of the GUF by the Monte Carlo Method [1].

\subsection{Multivariate models}

The GUM and Supplement 1 to the GUM are focused on measurement models having a single output quantity. However, many measurement problems have more than one output quantity depending on a common set of input quantities. A common case is in the electrical metrology when the measurand is complex. LNE-MCM software considers the case of multivariate models according to GUM Supplement 2. The two-dimensional coverage region considered in LNE-MCM software is an ellipse centred at the best estimates for the two involved output quantities.

\subsection{Sensitivity analysis}

Sensitivity analysis is used to determine how "sensitive" a model is to changes in the value of the parameters of the model. In the context of the evaluation of measurement uncertainty, it enables one to have a better knowledge on the contributions of the different input quantities to the variance of the output quantity (also called the uncertainty budget). Within the GUM Uncertainty Framework (GUF) this is provided by the partial derivatives of the measurement model, which is a local sensitivity analysis. On the contrary, there is no immediate counterpart of sensitivity with a Monte Carlo Method. The Supplement 1 to the GUM proposes to evaluate such contributions by holding all input quantities but one fixed at their best estimate and to perform a new Monte Carlo simulation for each input quantity. This method provides a quantification of the effect of the given input quantity on the standard deviation of the output quantity but is not computationally efficient for monotonic models since it requires additional simulations. Furthermore, when only one input quantity varies, holding all the other input quantities fixed at their best estimate, potential interaction effects cannot occur and will not be considered in the sensitivity analysis. To address this issue, we propose a global sensitivity analysis in LNE-MCM software based on the two following methods: the Spearman correlation coefficients for the monotonic measurement model and the Sobol' indices for more complex models. The main advantage of these two previous methods is that they are global in the sense that the sensitivity is investigated all over the range of the different input quantities whereas with the GUF only the sensitivity around the best estimate of the input quantities is considered. Thereafter, are briefly summarized the two methods. For more details we refer to [5]. In practice, for a sample of size $M$, we consider the ranks of each simulated value for the input $\mathrm{Xj}$ and for the output quantity $\mathrm{Yk}$. For each ranked pair $(\mathrm{Xj}, \mathrm{Yk})$, the difference di between the ranks is computed and the Spearman rank correlation coefficient between $\mathrm{Xj}$ and $\mathrm{Yk}$ is calculated as in (1)

$$
\rho_{j, k}=1-\frac{6 \sum_{i=1}^{M} d_{i}^{2}}{\left(M^{3}-M\right)}
$$

This correlation coefficient is normalized in order to obtain an estimation of the contribution of the input quantity $\mathrm{Xj}$ to the variance of the output quantity $\mathrm{Yk}$ according to (2):

$$
S_{j, k}=\frac{\rho_{j, k}^{2}}{\sum_{j=1}^{M} \rho_{j, k}^{2}}
$$

When the measurement model is not monotonic, Sobol' indices should be used. The Sobol' method is a global sensitivity analysis technique which determines the contribution of each input (or group of inputs) to the variance of the output. According the total variance decomposition theorem, the variance of an output 
quantity $\mathrm{Y}$ may be decomposed as the sum of the variance of the conditional expectation of $\mathrm{Y}$ given $\mathrm{Xi}$ and the expectation of the conditional variance of $\mathrm{Y}$ given $\mathrm{Xi}$ according to (3)

$$
V(Y)=V\left[E\left(Y \| X_{i}\right)\right]+E\left[V\left(Y \| X_{i}\right)\right]
$$

The first term $V_{i}=V\left[E\left(Y \| X_{i}\right)\right]$ denotes the part of the variance of $\mathrm{Y}$ due to the variations of the input quantity $\mathrm{Xi}$ while the second term denotes the part of the variance of $\mathrm{Y}$ that is due to all the input quantities but $\mathrm{Xi}$. The first order sensitivity index is then defined as (4)

$$
S_{i}=\frac{V_{i}}{V(Y)}
$$

A second order sensitivity index can also be obtained from the variance due to the couple of quantities $\mathrm{Xi}$ and $\mathrm{Xj}$ according to (5)

$$
S_{i, j}=\frac{V\left[E\left(Y \| X_{i}, X_{j}\right)\right]-V_{i}-V_{j}}{V(Y)}
$$

Higher order sensitivity indices can be obtained in the same manner, until $\mathrm{n}^{\text {th }}$ order (where $n$ is the number of input quantities). In LNE-MCM software, Sobol' indices are estimated by Monte Carlo Method, and available up to the second order. Consider two M-samples $\left(x_{i, j}\right)_{i=1, \ldots, n, j=1, \ldots, M}$ and $\left(x_{i, j}^{\prime}\right)_{i=1, \ldots, n ; j=1, \ldots, M}$. The first order sensitivity index is estimated by (6)

$$
\hat{S}_{i}=\frac{\hat{D}_{i}}{\hat{D}}
$$

and $\hat{D}_{i}$ and $\hat{D}$ are given by (7) and (8)

$$
\begin{gathered}
\hat{D}_{i}=\sum_{j=1}^{M} \frac{f\left(x_{1, j}, \ldots, x_{n, j}\right) f\left(x_{1, j}^{\prime}, \ldots, x_{(i-1), j}^{\prime}, x_{i, j}, x_{(i+1), j}^{\prime}, \ldots, x_{n, j}^{\prime}\right)}{M}-\hat{f}_{0}^{2} \\
\hat{D}=\frac{1}{M} \sum_{j=1}^{M} f^{2}\left(x_{1, j}, \ldots, x_{n, j}\right)-\hat{f}_{0}^{2}
\end{gathered}
$$

The quantity (9) denotes the empirical mean of $y$

$$
\hat{f}_{0}=\frac{1}{M} \sum_{j=1}^{M} f\left(x_{1, j}, \ldots, x_{n, j}\right)
$$

First $S_{i}$ and second order $S_{i, j}$ sensitivity indices provide then an uncertainty budget associated in terms of ranked contributions to the variance associated with the best estimate of the measurand.

\subsection{Validation of the GUM Uncertainty Framework using Monte Carlo Method}

The GUM Uncertainty Framework can be expected to work well in many circumstances. However, it is not always straightforward to determine whether all the conditions for its application hold. Since the domain of validity for the Monte Carlo Method is broader than the domain of the LPU method, it is recommended to use the first method. Nevertheless, it is often simpler to apply the LPU method described in the GUM. In this case, the assumptions for a valid application of the GUF should be verified by comparing the results obtained with the Monte Carlo Method and with the LPU. If the two results are similar, then the GUM Uncertainty Framework is considered to be valid. Otherwise, the Monte Carlo Method should be applied. The Supplement 1 to the GUM defines a criterion based on a comparison of the absolute differences of the respective endpoints of the coverage intervals obtained with the two methods with respect to a stipulated numerical tolerance. Let $u(y)=c \times 10^{l}$ be the standard deviation of the output quantity $y$ where $c$ is an $\mathrm{n}_{\text {dig }}$ decimal digit integer $\left(\mathrm{n}_{\mathrm{dig}}=1\right.$ or 2 if one wants to validate GUM method with 1 or 2 significant digit) then the numerical tolerance $\delta$ is given by $\delta=0.5 \times 10^{l}$. Let $\mathrm{d}_{\text {low }}$ and $\mathrm{d}_{\text {high }}$ be respectively the differences between the lower and upper bounds of the two coverage intervals given by (10) and (11)

$$
\begin{aligned}
& d_{\text {low }}=\left|y-U-y_{\text {low }}\right| \\
& d_{\text {high }}=\left|y+U-y_{\text {high }}\right|
\end{aligned}
$$

where $\mathrm{U}$ is the $95 \%$ expanded uncertainty given by the LPU method and $y_{\text {low }}, y_{\text {high }}$ are the bounds of the $95 \%$ coverage interval given by the Monte Carlo Method. If both $\mathrm{d}_{\text {low }}, \mathrm{d}_{\text {high }}$ are no larger than $\delta$, the comparison is favourable and the GUM Uncertainty Framework is considered to be validated. In LNE-MCM software, the user has to provide the result of the LPU method (best estimate, standard uncertainty, coverage factor) and $n_{\text {dig, }}$, and the validation is then automatically performed.

\subsection{Goodness of fit test}

The use of the Monte Carlo Method yields to a representation of the PDF associated with the output quantity. In some cases, the output quantity can be seen as an input quantity of another measurement model. To perform a new Monte Carlo simulation, it is necessary to determine the PDF of this output quantity. To this extent, goodness of fit test can be used to compare the empirical PDF resulting from the Monte Carlo simulation with the PDF from some usual probability distributions. First, within LNE-MCM, parameters of classical probability distributions (Gaussian, Student, lognormal, gamma, beta...) are provided by maximum likelihood estimation. Then Kolmogorov-Smirnov goodness of fit test is performed to select the theoretical distribution closest to the empirical one computed by Monte Carlo simulation.

\section{Conclusion}

To evaluate the measurement uncertainty with respect to the method described in the Supplement 1 to the GUM, a new validated software, called LNE-MCM, with a userfriendly interface will be made freely available online at the end of 2015. LNE-MCM software goes beyond the 
scope of the Monte Carlo Method, taking into account several output quantities, performing a sensitivity analysis to provide the uncertainty budget, validating the assumptions of the GUF and providing goodness of fit test in order to facilitate the use of an output quantity of a measurement model as an input quantity for another measurement model.

\section{References}

1. ISO/CEI GUIDE 98-3/S1:2008, Uncertainty of measurement - Part 3: guide to the expression of uncertainty in measurement (GUM:1995), Supplement 1: propagation of distributions using a Monte Carlo method, (2008)

2. ISO/CEI GUIDE 98-3:2008, Uncertainty of measurement - Part 3: guide to the expression of uncertainty in measurement (GUM :1995), (2008)

3. M. Matsumoto, T. Nishimura, Mersenne Twister: A 623-Dimensionally Equidistributed Uniform PseudoRandom Number Generator. ACM. Trans. Model. Comput. Simul. 8, 3-30 (1998)

4. ISO/CEI GUIDE 98-3/S1:2008, Uncertainty of measurement - Part 3: guide to the expression of uncertainty in measurement (GUM:1995), Supplement 2: extension any number of output quantities, (2011)

5. A. Saltelli, K. Chan, E.M. Scott, editors. Sensitivity Analysis, Wiley, (2000)

6. F.J. Massey, The Kolmogorov-Smirnov Test for Goodness of Fit. J. Am. Statist. Assoc. 46, 68-78, (1951)

7. A. Allard, N. Fischer, Recommended tools for sensitivity analysis associated to the evaluation of measurement uncertainty AMCTM IX, 1-12 (2012) 